\title{
MORBIDADE E MORTALIDADE HOSPITALAR EM RIBEIRÃO PRETO, SP (BRASIL), 1972 *
}

\author{
Juan Stuardo Yazlle Rocha**
}

\begin{abstract}
RSPU-B/353
Yazlle Rocha, J. S. Morbidade e mortalidade hospitalar em Ribeirão Preto, SP (Brasil), 1972. Rev. Saúde públ., S. Pallo, 11:214-28, 1977.

RESUMO: Estuda-se a morbidade e a morfalidade hospitalares na demanda de assistência médico-hospitalar do municipio de Ribeiräo Preto, ano de 1972. Destacaram-se pela sua frequiência e alta utilização de leitos, os grupos XI Complicações da Gravidez, Parto e Puerpério, VIII - Doenças do Aparelho Respiratório, VII - Doenças do Aparelho Circulatório, XVII - Acidentes, Envenenamentos e Violências, IX - Doença do Aparelho Digestivo e $X-$ Doenças do Aparelho Geniturinário. Estes grupos foram responsáveis por quase $60 \%$ da utilização de leitos hospitalares, pela população de Ribeirão Preto. Os grupos diagnósticos mais freqüêntes nos casos de obitos hospitalares foram: VII Doenças do Aparelho Circulatório, II - Tumores (Neoplasmas), VIII - Doenças do Aparelho Respiratório. I-Doenças Infecciosas e Parasitárias, XV - Certas Causas de Morbidade e Mortalidade Perinatais, XVII - Acidentes, Envenenamento "Violêtrcias.
\end{abstract}

Uniternos: Morbidade. Mortalidade. Hospitalizą̧äo, demanda. Assistência médica hospitalar.

A morbidade e a mortalidade de uma população são o preço pago pela mesma por viver como vive. A assistência médica à população (suas prioridades e mecanismos), é parte do mesmo estilo de vida '. As populações humanas têm experimentado intensas transformaçōes nas suas atividades de produção e nos seus padrões de doença e assistência médica, integrantes todos eles da mesma dinâmica social. Por isto "um numero maior e mais variado de medidas de morbidade é essencial para auxiliar na aplicação mais eficaz das técnicas e dos recursos disponiveis". (Logan $\left.{ }^{14}, 1964\right)$.

Entre os métodos de estudo da morbidade, baseados em registros contínuos, temos os estudos da demanda e/ou utilização de serviços de saúde.

A demanda não expressa todos os problemas de saúde da comunidade (e pode haver demanda sem "necessidade"), sendo ainda condicionada pelos sistemas de assistência médica vigentes (e seus fatores de coacão ou restrição). Porém, ela é mais

\footnotetext{
* Extraido da Tese de Doutoramento apresentada à Faculdade de Medicina de Ribeirảo Preto da USP, em 1974.

** Do Departamento de Medicina Social da Faculdade de Medicina de Ribeirão Preto da USP Rua Bernaldino de Campos, 1000 - Ribeirão Preto. SP — Brasil.
} 
YAZLLE ROCHA, J. S. - Morbidade e mortalidade hospitalar em Ribeirão Preto, SP (Brasil), 1972. Rev. Saúde públ., S. Paulo, 11:214-28, 1977.

objetiva e mais fácil de operacionalizar do que o conceito de necessidade, quando se trata de planejar recursos. Pode ser estudada a partir da utilização de serviços quando certas condições são satisfeitas (acessibilidade dos serviços a toda a população, adequação em qualidade e quantidade e relaçōes geográficas e funcionais entre os recursos e a população).

Nesta situação teremos que a utilização é igual à demanda, ou seja, que toda a demanda é satisfeita. A comprovação definitiva da satisfação da demanda só pode ser feita por investigação ao nível da comunidade, para verificar se toda procura de assistência foi atendida.

Ribeirão Preto $(S P)$, centro de assistência médico-hospitalar regional, reúne condições que nos permitem assegurar que praticamente toda a demanda de hospitalizações gerais do município, é satisfeita nele mesmo 19. 2". Conta ainda com um sistema de informática hospitalar que permite estudar as características da utilização ou demanda dos serviços hospitalares da cidade "'.

Em publicação anterior 20 expusemos a primeira parte do nosso estudo, apresentando, principalmente, a demanda de internação e a duração média das internações segundo idade e sexo.

O presente trabalho tem por objetivo estudar a morbidade e mortalidade hospitalar na demanda de hospitalizações gerais no municipio de Ribeirão) Preto, no ano de 1972.

\section{MATERIAL E METODOS}

$O$ material e métodos empregados foram descritos por ocasião da publicação, em 1975, da primeira parte deste estudo 20 Nessa ocasião foram estudados, também, os diagnósticos principal (definido como a causa da internação) e secundários (outros diagnósticos), e a condição de saída do paciente (vivo ou morto), a partir da folha de alta dos pacientes internados em 1972 nos hospitais gerais do município e proce- dentes de Ribeirão Preto. Os diagnósticos referidos ao Centro de Processamento de Dados Hospitalares (CPDH) são codificados segundo a Classificação Internacional de Doenças ( $8^{a}$ Revisão) ${ }^{16}$. Na verificação da consistência das informações, foram cotejados os diagnósticos do cartão perfurado com os constantes do prontuário do paciente, ao nível de especificação da lista de categorias de 3 dígitos da Classificação Interiacional de Doenças (CID). A tabulação dos diagnósticos foi feita segundo a lista A (Lista de 150 grupos) da CID, e mais tarde somados de forma a compor os 17 grupos da mesma classificação.

\section{RESULTADOS}

Em 1972 houve 40.832 internações (excluidos os recém-nascidos), nos hospitais gerais de Ribeirăo Preto, sendo 28.234 internações de pacientes do próprio município. Houve também 3.025 crianças recém-nascidas registradas nos hospitais e referidas ao $\mathrm{CPDH}$. A distribuição por idade e sexo, duração médias das hospitalizações, coeficientes de internação e indice de hospitalização para a população de Ribeirão Preto, já foram expostos em publicação anterior ${ }^{20}$.

A distribuição dos diagnósticos de internação (principal e secundário), os pacientes-dia e a duração média da internação, segundo os 17 grupos da CID e a chave $Y$, aparecem na Tabela 1.

A distribuição dos coeficientes por mil internações, da causa da internação, segundo os 17 grupos da CID, estão na Figura 1.

A Tabela 2 e a Figura 2 apresentam-nos a distribuição da morbidade hospitalar segundo os 17 grupos da CID e a chave $Y$, grupos etários, sexo e a duração média da internação.

A Tabela 3 e a Figura 3 apresentam-nos a distribuição de diagnósticos referidos em casos de óbitos hospitalares, segundo os 17 grupos da CID, grupo etário, sexo e duração média da internação. 
YAZLLE ROCHA, J. S. - Morbidade e mortalidade hospitalar em Ribeirão Preto, SP (Brasil), 1972. Rev. Saúde públ., S. Paulo, 11:214-28, 1977.

T A B E L A 1

Distribuição dos diagnósticos de internação (Principal e Secundários), pacientes-dia e duração média da internação, segundo os 17 grupos da CID* e o grupo $\mathrm{Y}^{* *}$ dos hospitais gerais de Ribeirão Preto, 1972.

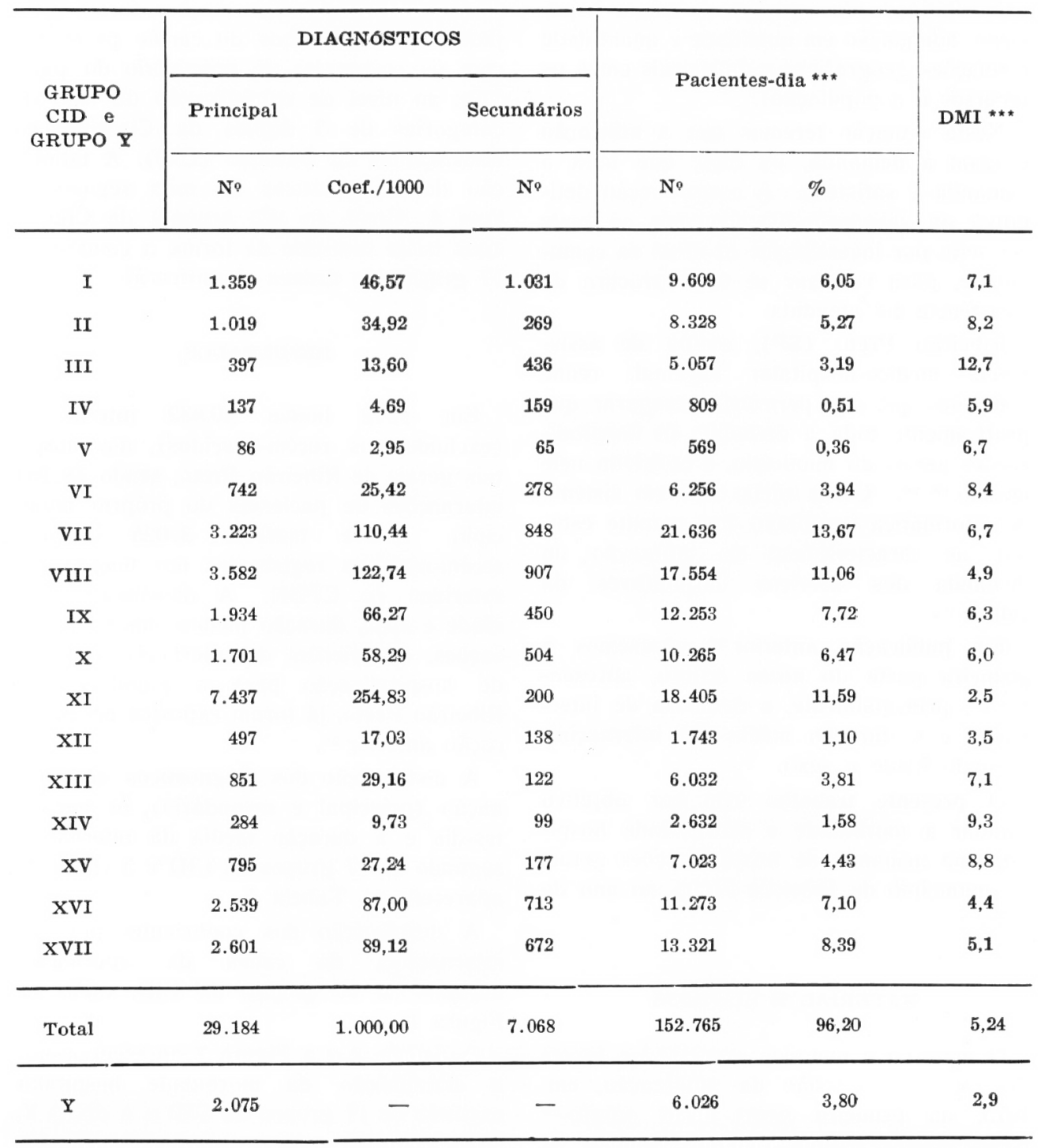

* Classificação Internacional de Doenças

* Classificações Suplementares da CID

*** Dos diagnósticos principais. 
YAZLLE ROCHA, J. S. - Morbidade e mortalidade hospitalar em Ribeirão Preto, SP (Brasil), 1972. Rev. Saúde públ., S. Paulo, 11:214-28, 1977.

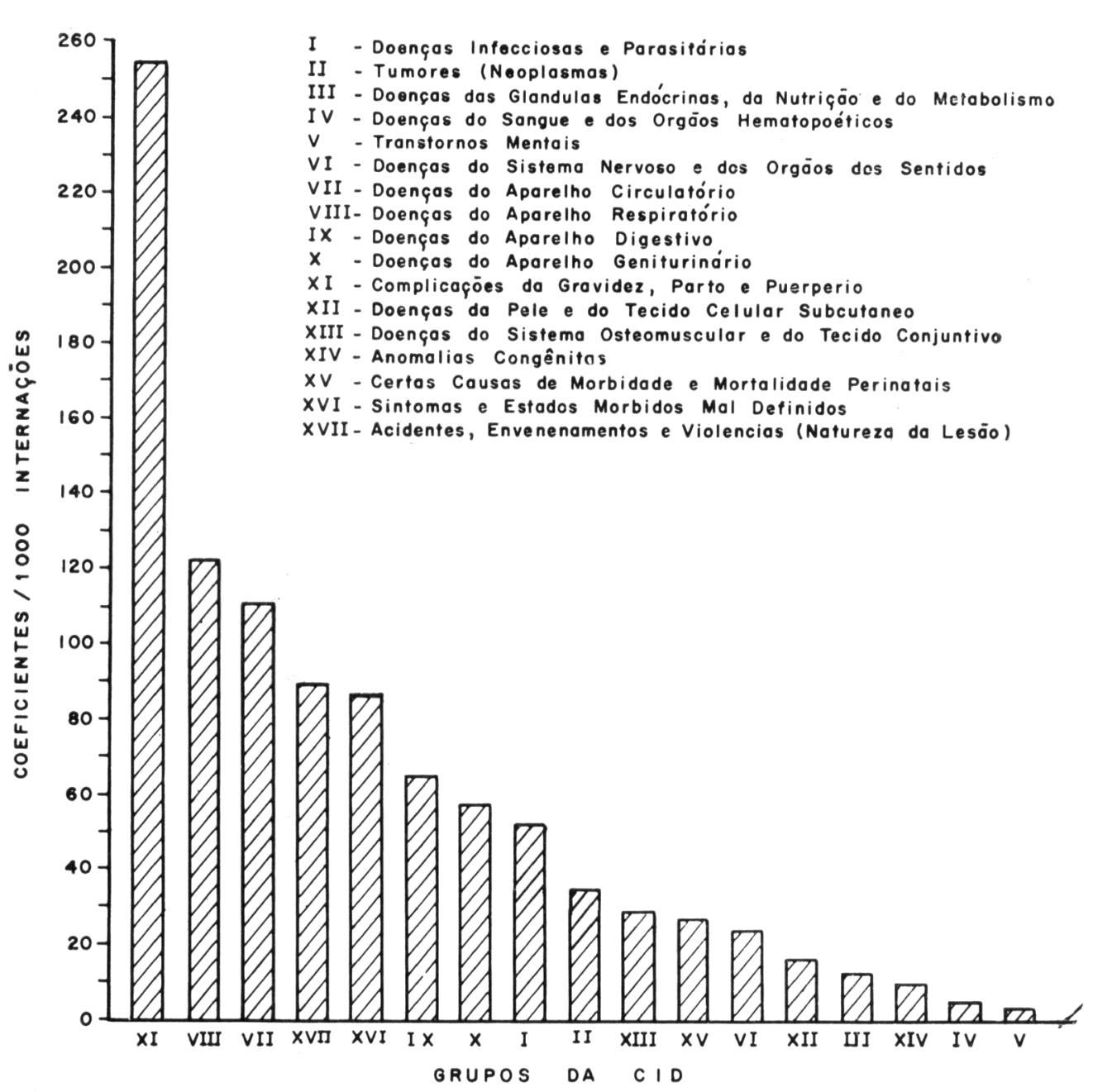

Fig. 1 - Coeficientes, por mil internações, da causa de internação, segundo os 17 grupos da CID, em hospitais gerais de Ribeirão Preto, 1972.

Todos estes dados serão comentados conjuntamente em ordem decrescente de freqüência.

Grupo XI - Complicações da gravidez, parto e puerpério.

É a mais freqüente causa de internação (254,83 casos/1.000 internações). Utilizou $11,59 \%$ dos leitos-dia ocupados, teve a menor duração média de internação, 2,5 dias (tabela 1, Figura 1). Foi mais freqüente nas pacientes de 15-34 anos (Tabela 2). Apareceu como causa de internação em $0,94 \%$ dos óbitos hospitalares (Tabela 3 ). Foi constituido principalmente dos grupos: A 118 - parto sem menção de complicações; A 117 - outras complicaçōes da gravidez, do parto e do puerpério e A 115 - outros abortos, ou não especificados. 


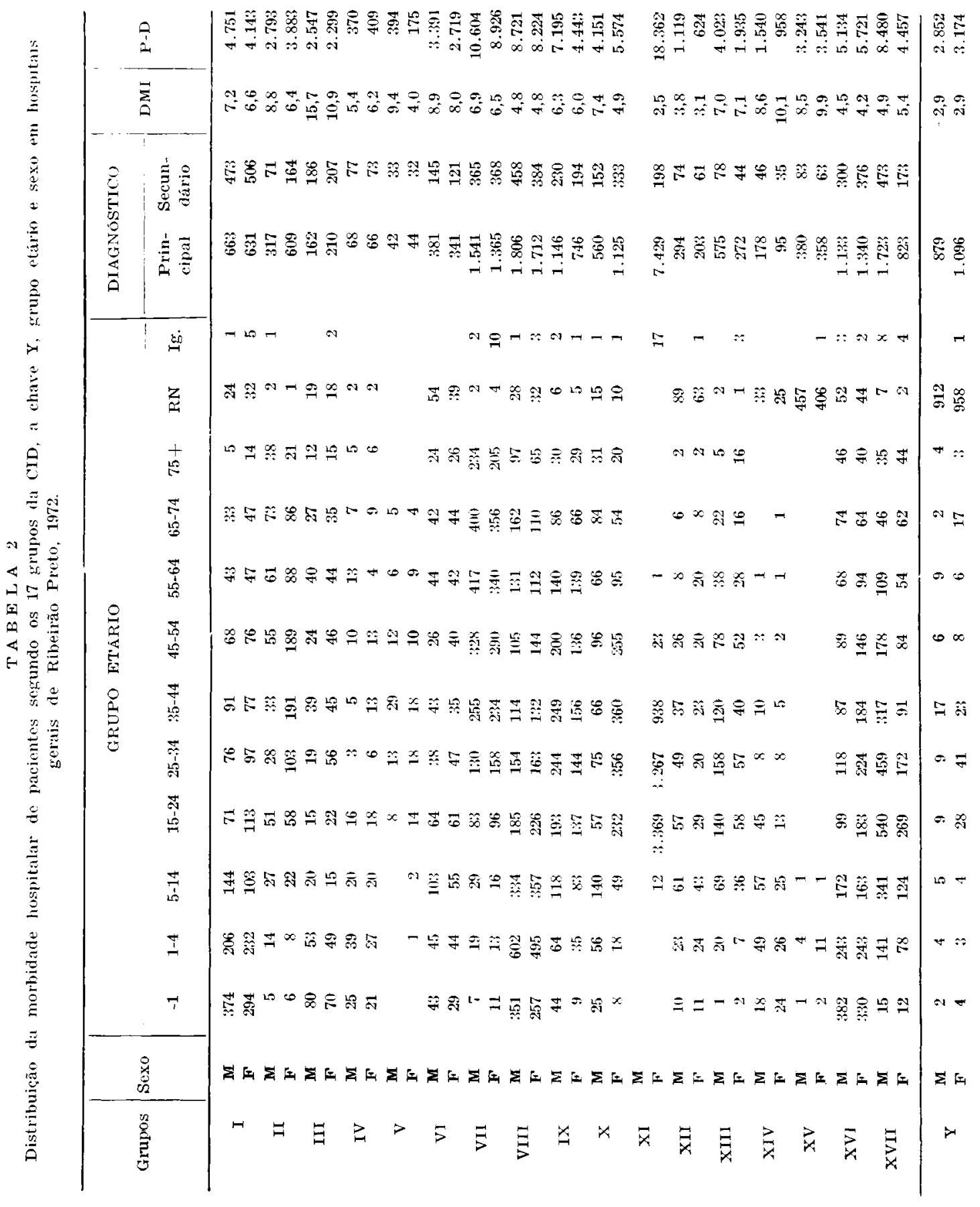


YAZLLE ROCHA, J. S. - Morbidade e mortalidade hospitalar em Ribeirão Preto, SP (Brasil), 1972. Rev. Saúde públ., S. Paulo, 11:214-28, 1977.

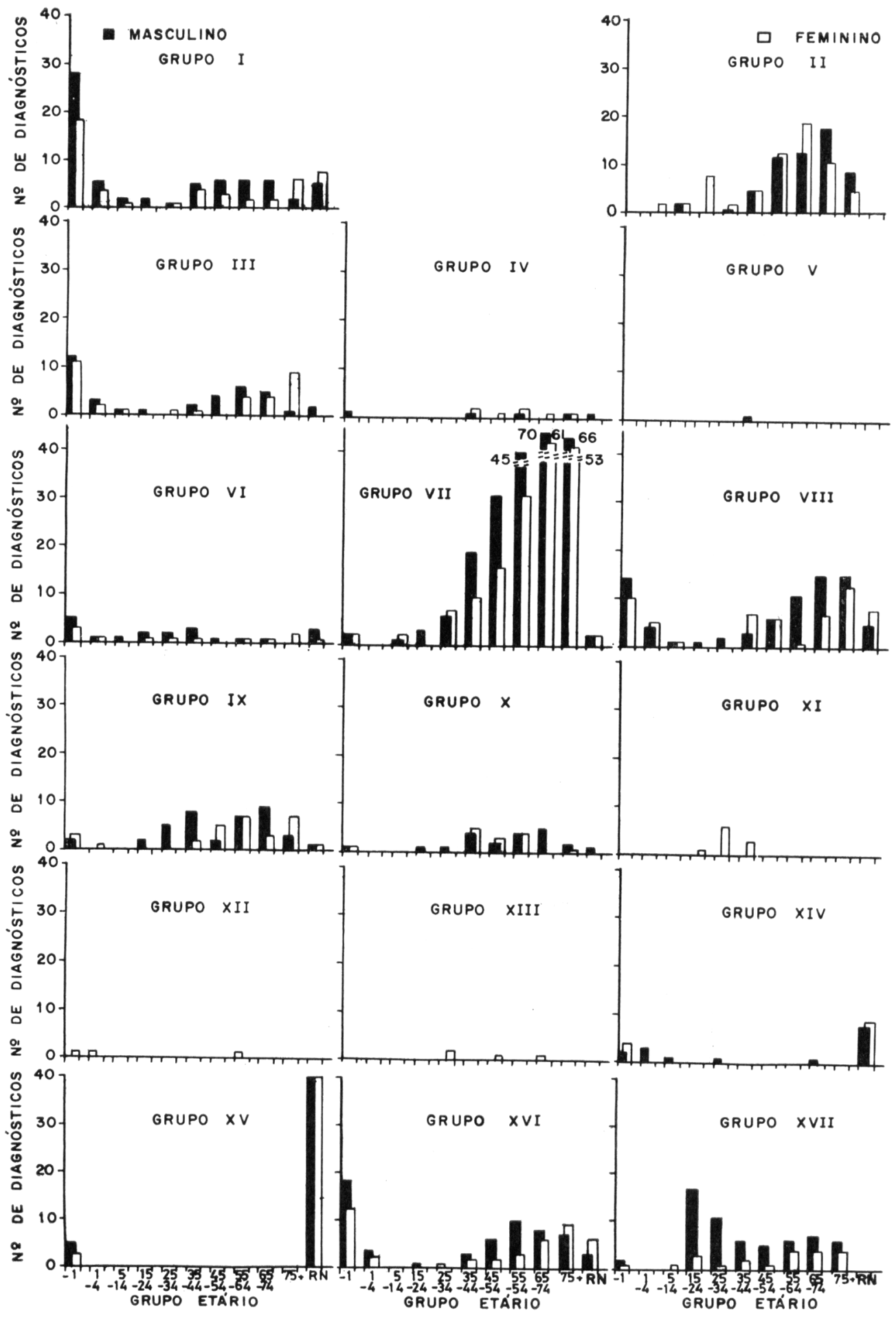

Fig. 2 - Distribuição da morbidade hospitalar de pacientes, segundo os 17 grupos da CID, o grupo $Y$, grupo etário e sexo, em hospitais gerais de Ribeirão Preto, 1972. 
YAZLLE ROCHA, J. S. - Morbidade e mortalidade hospitalar em Ribeirão Preto, SP (Brasil). 197z. Rev. Saude públ., S. Paulo, $11: 214-28,1977$.

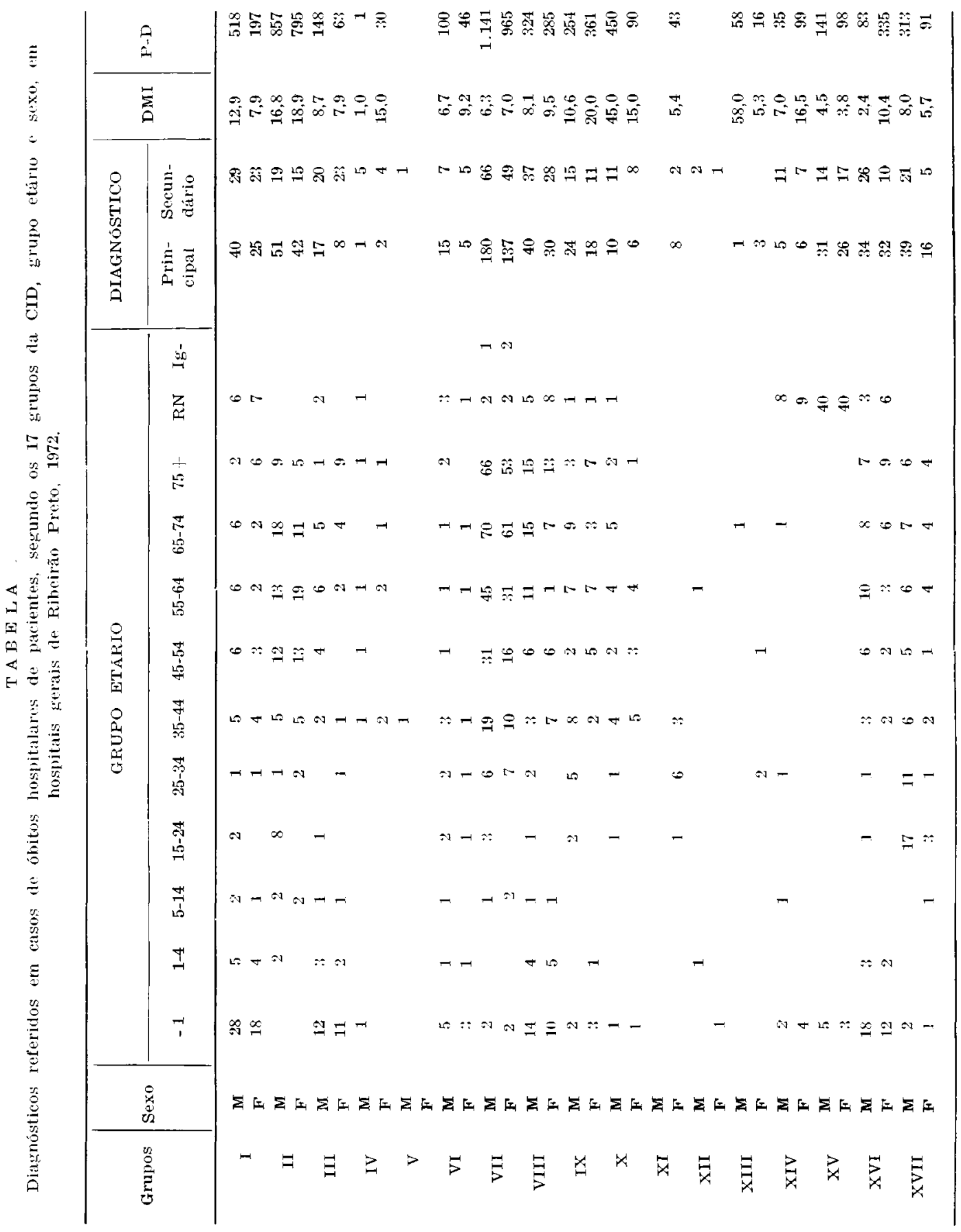


YAZLLE ROCHA, J. S. - Morbidade e mortalidade hospitalar em Ribeirão Preto, SP (Brasil), 1972. Rev. Saúde públ., S. Paulo, 11:214-28, 1977.

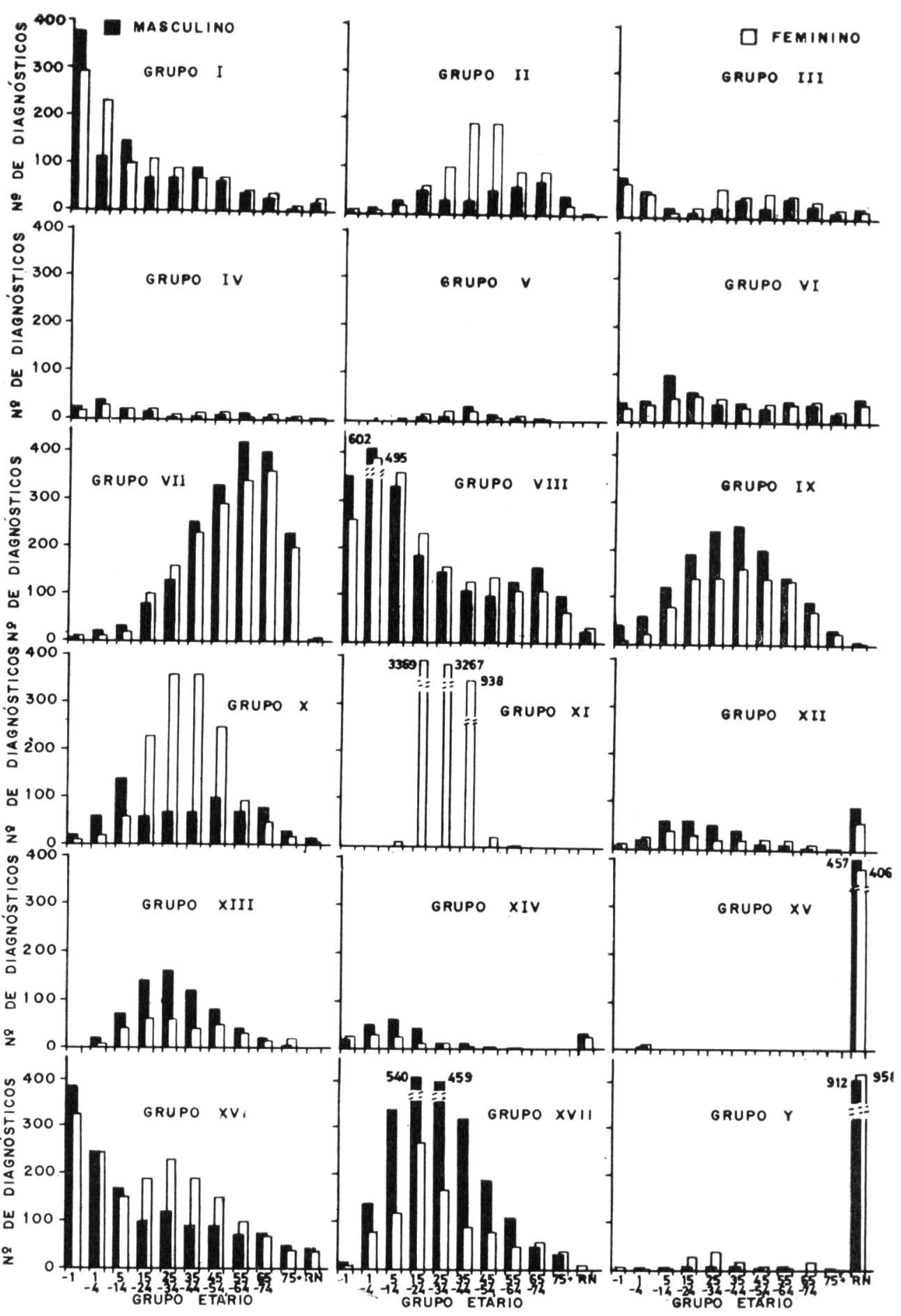

Fig. 3 - Diagnósticos referidos em casos de 6́bitos hospitalares de pacientes, segundo os 17 grupos da CID, grupo etário e sexo, em hospitais gerais de Ribeirão Preto, 1972. 
YAZLLE ROCHA, J. S. - Morbidade e mortalidade hospitalar em Ribeirâo Preto, SP (Brasil), 1975. Rer. Saúde públ., S. Paulo, 11:214-28, 1977.

$$
\begin{gathered}
\text { Grupo VIII - Doenças do aparelho } \\
\text { respiratório }
\end{gathered}
$$

Apareceu como causa da internação em 122,74 casos por mil internaçóes. Utilizou $11,06 \%$ dos leitos-dia ocupados. A duraçào média de internação foi de 4,9 dias (Tabela 1, Figura 1). Até os 5 anos e depois dos 55 anos predominou no sexo masculino, sendo pouco maior nesse intervalo entre as mulheres; foi mais freqüente nas internações na infância, culminando no grupo de 1-4 anos, decrescendo a seguir (Tabela 1, Figura 1). Apareceu como causa da internação de $8,22 \%$ dos óbitos hospitalares (Tabela 3), com ligeiro predomínio no sexo masculino. Foi mais freqüente nos casos de óbitos hospitalares no primeiro ano de vida e após os 55 anos de idade (Tabela 3, Figura 3). Constituiu-se principalmente dos grupos: A 92 - outras pneumonias; A 93 - bronquite, enfisema e asma e A 94 - hipertrofia das amidalas e vegetaçōes adenóides.

\section{Grapo VII - Doenças do aparelhı circulatório}

Foi o terceiro em treqüência, causando 110,44 de cada mil internaçóes, e primeiro em utilização de leitos hospitalares $(13,67 \%$ dos leitos-dia ocupados). A duração média de internação foi de 6,7 dias (Tabela 1, Figura 1). Foi mais frequiente no sexo masculino do que no feminino. Tornou-se mais freqüente a partir do grupo de 15-24 anos, culminando de 55-64 anos (Tabela 2, Figura 2). Apareceu como causa da internação em $37,21 \%$ dos casos de óbitos hospitalares. Predominou no sexo masculino (principalmente de 35-54 anos) en relação ao feminino. Atingiu a máxima frequêencia de 65-74 anos (Tabela 3, Figura 3). Constituilu-se principalmente dos grupos: A 84 - outras formas de doenças do coração; A 88 - outras doenças do aparelho circulatório e A 85 - doenças cérebro-vasculares.

\section{Grupo XVII - Acidentes, envenenamentos e violências (natureza da lesāo)}

Aparecell como causa da internação em 89,12 internações de cada mil. Causou a utilização de $8,30 \%$ dos leitos-dia ocupados. A duração média de internação foi de 5,1 dias (Tabela 1). Foi mais frequiente no sexo masculino, com freqüência máxima para ambos os sexos no grupo etário de 15-34 anos (Tabela 2, Figura 2). Foi causa da internação em $6,46 \%$ dos casos de óbitos hospitalares. Predominou no sexo masculino, tendo sua maior frequiência dos 15-34 anos (Tabela 3, Figura 3). Constituiu-se principalmente dos grupos: AN 145 - lacerações e ferimentos; AN 140 -. fratura dos membros e AN 143 - traumatismos intracranianos (exceto a fratura do crànio).

$$
\begin{aligned}
& \text { Grapo XVI - Sintomas e estados } \\
& \text { mórbidos mal definidos }
\end{aligned}
$$

Apareceu como causa de internação em 87,00 internaçīes de cada mil. Utilizou $7,10 \%$ dos leitos-dia ocupados. A duração média de internação foi de 4,4 dias (Tabela 1). Foi mais frequiente até os 5 anos, decrescendo com a idade. Dos 15-64 anos predominou no sexo feminino (Tabela 2, Figura 2). Foi causa de internação em $7,75 \%$ dos casos de óbitos hospitalares, onde predominou no sexo masculino (com exceção dos grupos 75 anos ou mais $e$ recém-nascidos), (tabela 3 , figura 3 ). Constituiu-se principalmente de sintomas relatiros aus aparelhos ou orgãos do corpo humano (780-789 da lista de categorias de 3 algarismos da $\mathrm{CID})(\mathrm{CPDH}+1972)$.

\section{Grupo $I X$ - Doenças do aparelho digestivo}

Apareceu como causa da internação em 66,27 de cada mil internaçōes. Foj responsável por $7,72 \%$ dos leitos-dia ocupados. A duração média de internação foi de 6,3 dias (Tabela 1). Predominou no sexo masculino, sendo de frequiência crescente desde 
YAZLLE ROCHA, J. S. - Morbidade e mortalidade hospitalar em Ribeirão Preto, SP (Brasil), 1972. Rev. Saúde pübl., S. Paulo, 11:214-28, 1977.

o primeiro ano de idade até os 35-44 anos para decrescer até o fim da vida (Tabela 2, Figura 2). Apareceu como causa da internação em 4,93\% dos casos de óbitos hospitalares, apresentando-se, de maneira geral, predominante no sexo masculino e crescente com a idade (Tabela 3, Figura 3). Constituiu-se principalmente dos grupos: A 101 - obstrução intestinal e hérnia; A 104 - outras doenças do aparelho digestivo e A 98 - úlcera péptica.

$$
\begin{aligned}
\text { Grupo } X- & \begin{array}{l}
\text { Doenças do aparelho } \\
\text { geniturinário }
\end{array}
\end{aligned}
$$

Apareceu como causa da internação em 58,29 internações de cada mil. Foi responsável por $6,47 \%$ dos leitos-dia ocupados. A duração média de internação foi de 6,0 dias (Tabela 1). Até os 14 anos teve predomínio no sexo masculino. Dos 15-64 anos predominou no sexo feminino, atingindo a frequiência máxima aos 25-44 anos. Dos 65 anos em diante foi mais frequiente no sexo masculino (Tabela 2, Figura 2). Foi causa da internação de $1,88 \%$ dos óbitos hospitalares. Nestes casos, foi mais freqüente entre 35 e 64 anos (Tabela 3, Figura 3). Constituiu-se principalmente dos grupos: A 111 - outras doenças do aparelho geniturinário; A 110 - doenças da mama e A 108 - cálculos do aparelho urinário (com maior freqüência no sexo masculino).

$$
\begin{gathered}
\text { Grupo I - Doenças infecciosas e } \\
\text { parasitárias }
\end{gathered}
$$

Apareceu como causa da internação em 46,57 internações de cada mil. Utilizou $4,20 \%$ dos leitos-dia ocupados. A duração média da internação foi de 7,1 dias (Tabela 1). Mais freqüente no primeiro ano de vida, decresceu com o aumento da idade, ora predominando ligeiramente no sexo masculino ora no feminino (Tabela 2, Figura 2). Apareceu como causa de internação em $7,63 \%$ dos casos de óbitos hospitalares. Nestes, foi mais freqüente no primeiro ano de vida, e dos 35-74 anos, predominando no sexo masculino (Tabela 2,
Figura 2). Constituiu-se principalmente dos grupos: A 5 - enterite e outras doenças diarréicas; A 44 - todas as demais doenças classificadas como infecciosas e parasitárias e A 32 - tripanossomíase.

\section{Grapo $I I$ - Tumores}

Apareceu como causa da internaçãu em 34,92 internações de cada mil. "Consumiu" $5,27 \%$ dos leitos-dia ocupados naquele ano. A duração média de internação foi de 8,2 dias (Tabela 1). Dos 15-74 anos foi mais freqüente no sexo feminino, atingindo o máximo dos 35-54 anos (Tabela 2, Figura 2). Apareceu como causa da internaçāo em $10,92 \%$ dos casos de óbitos hospitalares. Nestes, tornou-se crescente a partir do grupo 35-44 anos, para cair de frequêencia após os 74 anos. No sexo masculino a freqüência máxima foi de 65-74 anos, no sexo feminino de 55-64 anos (Tabela 3, Figura 3). Constituiu-se principalmente dos grupos: A 61 - tumores benignos e tumores de natureza não especificada; A 58 tumor maligno de outras localizações e de localizações não especificadas (em todos estes grupos foi mais frequiente no sexo feminino); A 55 - tumor maligno do colo do útero e A 47 - tumor maligno do estômago (predominando, este, no sexo masculino).

$$
\begin{aligned}
\text { Grupo XIII - } & \text { Doenças do sistema } \\
& \text { osteomuscular e do } \\
& \text { tecido conjuntivo }
\end{aligned}
$$

Apareceu como causa da internação em 29,16 internações de cada mil. "Consumiu" $3,81 \%$ dos leitos-dia ocupados. A duração média da internação foi de 7,1 dias (Tabela 1). Foi mais frequiente no sexo masculino do que no feminino. Crescente após o primeiro ano de vida, atingiu sua máxima frequiência no grupo de 25-34 anos, decrescendo após este grupo etário (Tabela 2, Figura 2). Foi causa de internação de $0,47 \%$ dos casos de óbitos hospitalares (Tabela 3). Constituiu-se principalmente dos grupos: A 125 - outras doenças do 
YAZLLE ROCHA, J. S. - Morbidade e mortalidade hospitalar em Ribeirão Preto, SP (Brasil), 1972. Rev. Saúde pübl., S. Paulo, 11:214-28, 1977.

sistema esteomuscular e do tecido conjuntivo; A 121 - artrite e espondilite e A 123 - Osteomielite e periostite (no primeiro e último destes grupos, A 125 e A 123, com amplo predomínio no sexo masculino).

Grupo $X V$ - Certas causas de morbidade e mortalidade perinatais

Apareceu como causa da internação em 27,24 internações de cada mil e utilizou $4,43 \%$ dos leitos-dia ocupados naquele ano. A duração média de internação foi de 8,8 dias (Tabela 1). Esteve presente entre os recém-nascidos (um pouco maior no sexo masculino do que no feminino) e crianças até os 4 anos de idade (predomínio no sexo feminino - tabela 2, Figura 2). Apareceu como causa da internação em $6,69 \%$ dos casos de óbitos hospitalares, com distribuição semelhante à descrita para a morbidade (Tabela 3, Figura 3). Constituiu-se principalmente dos grupos: A 135 - outras causas de morbidade e de mortalidade perinatais; A 134 - afeç̧ões anóxicas e hipóxicas não classificadas em outra parte $e$ A 131 - lesões ao nascer e partos distócicos.

$$
\begin{array}{r}
\text { Grupo VI - Doenças do sistema nervoso } \\
\text { e dos órgãos dos sentidos }
\end{array}
$$

Apareceu como causa da internação em 25,42 internações de cada mil e utilizou $3,94 \%$ dos leitos-dia ocupados. A duração média de internação foi de 8,4 dias (Tabela 1). Sem muitas variações pelos grupos etários, apresentou maior freqüência no sexo masculino, no grupo de 5-24 anos (Tabela 2, Figura 2). Apareceu como causa da internação em $2,35 \%$ dos casos de óbitos hospitalares, sendo mais freqüente no sexo masculino (Tabela 3, Figura 3). Constituiu-se principalmente dos grupos: A $79-$ outras doenças do sistema nervoso e dos órgãos dos sentidos; A 72 - meningite e A 76 - catarata.

\section{Grupo XII - Doenças da pele e do tecido} celular subcutâneo

Foi causa da internação de 17,03 de cada mil internações e utilizou $1,10 \%$ do total de leitos-dia ocupados. A duração média de internação foi de 3,5 dias (Tabela 1). Predominou no sexo masculino sobre o feminino, e alcançou maior freqüência no grupo de recém-nascidos e no grupo de 5-24 anos (Tabela 2, Figura 2). Não foi causa de internação de nenhum caso de óbito hospitalar (Tabela 3). Constituiu-se principalmente do grupo A 119 - infecções da pele e do tecido celular subcutâneo (com predomínio do sexo masculino sobre o feminino).

$$
\begin{aligned}
\text { Grupo } I I I- & \text { Doenças das glândulas } \\
& \text { endócrinas, da nutrição e } \\
& \text { do metabolismo }
\end{aligned}
$$

Foi causa da internação de 13,60 de cada mil internações. Foi mais freqüente como diagnóstico secundário que como causa de internação. Utilizou 3,19\% dos leitos-dia ocupados naquele ano. A duração média da internação por este grupo foi a maior de todos os grupos: 12,7 dias (Tabela 1). Mais freqüente no primeiro ano de vida, decresceu até os 14 anos (predominando no sexo masculino), para aumentar de freqüência a partir dos 15 anos (predominando no sexo feminino), com freqüência máxima no grupo de 25-34 anos (Tabela 2, Figura 2). Foi causa de internação em $2,93 \%$ dos casos de óbitos hospitalares, com maior freqüência no primeiro ano de vida (Tabela 3 , Figura 3). Constituiu-se principalmente dos grupos: A 65 - avitaminoses e outras deficiências nutricionais e A 64 - diabetes mellitus.

\section{Grupo XIV - Anomalias congênitas}

Foi causa da internação em 9,73 de cada mil internações. Utilizou-se $1,58 \%$ dos leitos-dia ocupados. A duração média de internação foi de 9,3 dias (Tabela 1). Predominou no sexo masculino, de 1-24 anos, e entre os recém-nascidos (Tabela 2, Figura 
YAZLLE ROCHA, J. S. - Morbidade e mortalidade hospitalar em Ribeirão Preto, SP (Brasil), 197E. Rev. Saúde públ., S. Paulo, 11:214-28, 1977.

2). Foi causa da internação de $0,13 \%$ dos casos de óbitos hospitalares, com maior frequiência no grupo de recém-nascidos (Tabela 3, Figura 3). Constituiu-se principalmente dos grupos: A 130 - as demais anomalias congênitas e A 127 - anomalias congênitas do coração.

\section{Grupo IV - Doenças do sangue e dos órgãos dos sentidos}

Foi causa da internação de 4,69 de cada mil internações e mais freqüente como diagnóstico secundário que como causa da internação. Utilizou $0,51 \%$ do total de leitos-dia ocupados. A duração média das internaçōes foi de 5,9 dias (Tabela 1). Com poucas oscilações de acordo à idade, foi mais freyüente entre o primeiro ano de vida e os 14 anos. Dos 15-44 anos foi mais freqüente no sexo feminino (Tabela 2, Figura 2). Apareceu como causa da internação em $0,35 \%$ dos casos de óbito hospitalar (Tabela 3). Constituiu-se principalmente do grupo A 67 - anemias.

\section{Grupo V - Transtornos mentais}

Foi causa da internação de 2,95 internaçōes de cada mil. "Consumiu" $0,36 \%$ dos leitos-dia ocupados naquele ano. A duração média das internações foi de 6,7 dias (Tabela 1). Apresentou maior freqüência de casos no grupo de 35-44 anos (Tabela 2). Não foi causa de internação de nenhum óbito hospitalar (Tabela 3). Constituitl-se principalmente de casos do grupo A $70-$ neurose, transtornos da personalidade $e$ outros transtornos mentais não psicóticos.

Grupo $Y$ - Classificações suplementares

Equivalente em freqüência a $7,1 \%$ das internações, utilizaram $3,80 \%$ dos leitos-dia ocupados no ano de 1972, com uma duração média por internação de 2,9 dias. Constituiu-se principalmente de recém-nascidos nos hospitais gerais e que ali permaneceram durante alguns dias.

\section{DISCUSSÃO}

O sistema de informação utilizado ( $\mathrm{CPDH}$ do Departamento Médico Social) foi avaliado quanto à cobertura e consistência das informações, e os resultados foram apresentados no nosso trabalho anteriormente publicado $\div$ ".

Quanto à qualidade das informaçōes médicas do prontuário, algumas considerações são necessárias. É sabido que às vezes não é possivel se fazer um diagnóstico preciso perante determinados quadros clínicos, e que muitas vezes o médico, de boa fé, erra determinados diagnósticos. Não pretendemos, por isso, defender que os diagnósticos referidos ao $\mathrm{CPDH}$, sejam diagnósticos de certeza, e nem é esse o problema que aqui queremos estudar. Seria melhor, do ponto de vista da atenção médica, e das estatísticas de morbidade, que o médico não errasse nunca, mas isto ainda não é a realidade.

No entanto, acreditamos que o número de acertos supere amplamente o número de erros cometidos (do contrário a medicina estaria em posição insustentável). O que nos interessa neste estudo, é que após o diagnóstico, ou a impressão diagnóstica do médico, a conduta a seguir decorre da idéia que ele faz do problema do doente, e os recursos para a saúde são consumidos segundo as impressōes que os médicos têm dos problemas dos seus pacientes. O diagnóstico, ou a impressão diagnóstica, a conduta, e a evolução posterior do paciente, ficam registrados pelo médico no prontuário do paciente. É preciso que o sistema de informação reflita 0 que é registrado no prontuário médico de cada paciente, no sentido de que a informação escrita na folha de egresso, codificada e perfurada, se mantenha em concordância com o que foi registrado pelo médico.

Os diagnósticos processados pelo $\mathrm{CPDH}$ foram confrontados com os diagnósticos registrados no prontuário do paciente e a consistência* encontrada oscilou entre

\footnotetext{
* Definimos a consistência como a percentagem de concordância entre as informações do cartão perfurado e as contidas no prontuá rio do paciente.
} 
YAZLLE ROCHA, J. S. - Morbidade e mortalidade hospitalar em Ribeirão Preto. SP (Brasil), 1972. Rev. Saude públ., S. Paulo, 11:214-28, 1977.

89 e $97 \%$. Como neste trabalho utilizamos grupamentos diagnósticos (Lista A e os 17 grupos da CID), a consistência dos dados que apresentamos deve ser maior que a acima referida.

Consideramos, pois, nosso sistema de informaçōes eficaz quanto à cobertura e consistència das informações.

\section{CAUSAS DAS INTERNAÇOES}

A utilização dos leitos hospitalares é condicionada, também, ${ }^{2,13}$ pelos tipos de problemas de saúde da população que requerem hospitalização; a composição relativa destes problemas, como seria de esperar, varia muito entre localidades e paises, e mesmo dentro de um país ao longo dos anos (OPASt, 1966; OPAS', 1970; OPAS", 1974; Ashley", 1972). O estudo da composição da demanda de hospitalização, segundo os 17 grupos da CID, tem aumentado de interesse nos últimos tempos com o surgimento de técnicas de planejamento que levam em consideração estes dados (Miller 1i, 1970; Alvim 1, 1971). Analisando a composição relativa das causas de internação segundo os 17 grupos da CID na

Ribeirão Preto
Venezuela
Costa Rica
El Salrador.
Honduras

$\begin{array}{rr}\mathrm{XI} & \text { VIII } \\ \mathrm{XI} & \mathrm{I} \\ \mathrm{XI} & \mathrm{I} \\ \mathrm{XI} & \mathrm{I} \\ \mathrm{XI} & \mathrm{I}\end{array}$

A semelhança no padrão de morbidade hospitalar entre os paises é evidente; na comparação com a morbidade hospitalar de Ribeirão Preto a semelhança é grande, havendo como discrepância principal, a inclusão do grupo VII - doenças do aparelho circulatório, entre os 6 primeiros grupos, e a defasagem do grupo 1 - doenças infecciosas e parasitárias ao sétimo lugar. A defasagem explica-se, em parte. porque em Ribeirão Preto, muitos casos são referidos ao CPDH como "desidratação" SAI, sendo portanto incluidos no grupo XVI - sintomas a estados mórbidos mal definidos. (Quanto ao grupo VII, outros trabathos têm apontado a importância das cardio- demanda de hospitalização de Ribeirão Preto (Tabelas 1, 2, 3), vemos que há grupos que dão grande utilização pelo número elevado de internaçóes que apresentam, embora a duração média delas seja baixa (Tabela 1, grupo XI); outros há que participando con uma proporção menor de internaçōes, por causa das internações prolongadas que ocasionam, dão uma utilização apreciável (Tabela 1, grupos 1, Il e IX); consideramos também como muito importantes os grupos de elevada freqüência e duração média de internaçóes (Tabela 1, grupos VII, XVII). Para Ribeirão Preto, em 1972, os grupos VII, XI, VIII, XVIl e IX, somados, consumiram mais da metade dos leitos-dia utilizados pela população naquele ano. $\mathrm{O}$ predomínio de alguns grupos da CID sobre outros, como causas de internação, determina o padrão de internação de uma localidade ou país. Baseados nos dados de OPAS (1974)", o coeficiente por mil internações dos 17 grupos da CID, em ordem decrescente de frequiencia dos 6 primeiros grupos, e comparando com os resultados de Ribeirão Preto, (excluindo o grupo XVI - Sintomas e Estados Morbidos Mal Definidos) temus:

$\begin{array}{rrrrr}\text { VII } & \text { XVII } & I X & X & \text { (I) } \\ \text { VIII } & \text { XVII } & I X & X & \\ \text { VIII } & \text { XVII } & I X & X & \\ \text { XVII } & \text { VIII } & X & I X & \\ \text { XVII } & \text { VIII } & \text { IX } & X\end{array}$

patias em Ribeirão Preto 11.1s. Um achado interessante, neste trabalho, foi o elevado coeficiente por mil internações, de doenças do aparelho respiratório, (grupo VIII), encontrado em Ribeirão Preto, terra quente, de inverno não rigoroso e carente da poluição atmosférica de centros mais industrializados, causas estas geralmente apontadas como responsáveis pela maior frequiência de afecçijes respiratórias.

O comportamento dos grupos da CID, especialmente os VIII, VII, I, XVII, II, segundo idade e sexo (Figura 2), tem características concordantes com os padrões epidemiológicos já descritos $\cdots 1 \cdots, 1 \%$, o que vem confirmar que a utilização de leitos em 
YAZLLE ROCHA, J. S. - Morbidade e mortalidade hospitalar em Ribeirão Preto, SP (Brasil), 1972. Rev. Saúde públ., S. Paulo, 11:214-28, 1977.

Ribeirão Preto é praticamente a demanda de assistência médico-hospitalar do município, e que a demanda (apesar dos fatores de coação ou restrição do sistema de assistência médica) reflete com bastante aproximação a morbidade da comunidade, que exige cuidados hospitalares.

A pesquisa dos fatores que determinam o padrão de morbidade de uma comunidade é objeto da epidemiologia. A quantificação e o estudo da composição relativa da demanda são importantes, também, porque isto permitirá adequar os recursos da assistência médica às necessidades (ou à demanda). O estudo da utilização de recursos (no caso, os leitos hospitalares), nos permitirá planejar o impacto das ações de saúde, no sentido de que diminuindo ou controlando determinados problemas de saúde, seriam liberados os recursos por eles absorvidos, de forma a poder empregá-los no ataque a outros problemas de saúde.

\section{CON CLUSOES}

1. Em Ribeirão Preto, no ano de 1972 , destacaram-se pela frequiência entre o total de hospitalização, os seguintes grupos de doenças:

$$
\begin{aligned}
& \mathrm{XI} \text { - Complicações da gravidez, } \\
& \text { parto e puerpério } \\
& \text { VIII - } \text { Doenças do aparelho respira- } \\
& \text { tório } \\
& \text { VII - } \text { Doenças do aparelho circula- } \\
& \text { tório } \\
& \text { XVII - } \text { Acidentes, envenenamentos e } \\
& \text { violências } \\
& \text { IX - Doenças do aparelho digestivo } \\
& \text { X - Doenças do aparelho genitu- } \\
& \text { rinário. }
\end{aligned}
$$

2. Estes grupos foram responsáveis por quase $60 \%$ da utilização de leitos hospitalares gerais, pela população do municipio de Ribeirão Preto.

3. Os grupos de doenças mais freqüentes entre os casos de óbitos hospitalares foram:

$$
\begin{aligned}
& \text { VII - Doenças do aparelho circula- } \\
& \text { II - Tumores (neoplasmas) } \\
& \text { VIII - Doenças do aparelho respira- }
\end{aligned}
$$

YAzLle Rocha, J. S. [Hospital mortality and morbidity in Ribeirão Preto, State of S. Pattlo (Brazil), 1972.] Rev. Saúde públ., S. Patlo, $11: 214-28.1977$.

ABSTRACT: Hospital Morbidity and Mortality in general hospital beds' demands from the county of Ribeirão Preto in 1972 were studied. The main groups by frequency and hospital beds' utilization were: XI - Complications of Pregnacy, Childbirth, and Puerperium, VIII - Diseases of the Respiratory System, VII - Disease of the Circulatory System, XVII - Accidents, Poisonings, and Violence, $I X$ - Disease of the Digestive System and $X$ - Disease of the Genitourinary System. These groups were responsible for almost sixty per cent of hospital beds' atilization. The main groups in hospitalar death cases were: VII Disease of the Circulatory System, II - Neoplasms, VIII - Disease of the Respiratory System, I - Infective and Parasitic Disease, $X V$ - Certain Causes of Perinatal Morbidity and Mortality, XVII - Accidents, Poisonings, and Violence.

UNITERMS: Morbidity. Mortality. Hospitalization, demand. Hospital medical assistance. 
YAZLLE ROCHA, J. S. - Morbidade e mortalidade hospitalar em Ribeirão Preto, SP (Brasil), 1972. Rev. Saúde públ., S. Paulo, 11:214-28, 1977.

\section{REFERENCIAS BIBLIOGRAFICAS}

1. ALVIM, E. F. Técnica de programación integrada de salud. Bol. Ofic. sanit. panamer, 71:469-80, 1971.

2. ANDERSON, D. O. Measurement of use and demand: uses of epidemiology in planning health services. [Paper presented at the International Scientific Meeting of the International Epidemiological Association, 6th, Belgrade, Yugoslávia, 1973].

3. ASHLEY, J. S. A. Present state of statistics from hospital in patient data and their uses. Brit. J. prev. soc, Med., 26 :135-47, 1972.

4. Centro DE Processamento DE DADOS. Relatório. Ribeirão Preto, 1972.

5. CLARK, D. W. \& MACMAHON, B., ed. Preventive Medicine. Boston, Little, Brown, 1967.

6. Las CONDICIONES DE SALUD EN LAS AMERICAS: 1961-1964. (Organización Panamericana de la Salud) Washington, D. C., 1966. (OPAS - Publ. cient., 138).

7. GAETE, J. et al. Apuntes sobre ciencias sociales y salud, Costa Rica, C. A., Consejo Superior de la Universtad de Centro America. (Cuadernos de Ciencias de la Salud).

8. LaS CONDICIONES DE SALUD EN LAS AMERICAS: 1965-1968. (Organización Panamericana de la Salud) Washington, D. C., 1970. (OPAS - Publ. cient., 207).

9. Las CONDICIONES DE SALUD EN LAS AMERICAS : 1969-1972. (Organización Panamericana de la Salud) Washington, D. C., 1974. (OPAS-Publ. cient. 287).

10. FAVERO, M. et al. Organização de um centro de informática hospitalar em nivel local. Rev. paul. Hosp., 21:151-7, 1973.

11. HADDAD, N. Inquérito epidemiológico sobre cardiopatias crônicas em um bairro de Ribeirão Preto, São Paulo, Brasil. Arq. Fig. S. Paulo, 32/33: 27-77, $1967 / 1968$.
12. HOBSON, W., ed. The theory and practice of public health. 4th ed. London, Oxford University Press, 1975.

13. LLEWELYN-DAVIES, R. \& MACAULAY, H. M. C. Planificación y administracion de hospitales. Washingtion, D. C., Organización Panamericana de la Salud, 1969. (OPAS - Publ. cient, 191).

14. LOGAN, R, F. L. Assessment of sickness and health in the community: needs and methods. Med. Care, 2:173-90; 218-25, 1964.

15. MACMAHON, B. \& PUGH, T. F. Epidemiology principles and methods. Boston, Little, Brown, 1970.

16. MANUAL da Classificação Estatística Internacional de Doenças, Lesōes e Causas de óbito. Washington, D. C., Organización Panamericana de la Salud, 1969. (OPAS - Publ. cient., 190).

17. MILlER, J. E. An indication to aid management in assigning program priorities, Publ-Hlth, Rep., 85: 725-31, 1970.

18. PUFFER, R. R. \& GIFFITH, G. W Caracteristicas de la mortalidad urbana. Washington, D. C., Organizacion Panamericana de la Salud, 1968. (OPAS - Publ. cient., 151).

19. YAZLLE ROCHA, J, S. Estudo da utilizaça do leitos hospitalares gerais do município de Ribeirão Preto, pela po. pulaçâo do municipio, no ano de 1972. Ribeirão Preto, S.P., 1974. [Tese de Doutoramento - Faculdade de Medicina de Ribeirão Preto da Universidade de São Paulo].

20. YAZLLE ROCHA, J. S. Utilização de leitos hospitalares gerais em Ribeirão Preto, São Paulo (Brasil). Rev. Saude puibl., S. Paulo, 9:477-93, 1975.

Recebiäo para publicação em 30/08/1976 Aprovado para publicação em 17/12/1976 